\title{
LYAPUNOV GRAPHS AND FLOWS ON SURFACES
}

\author{
K. A. DE REZENDE AND R. D. FRANZOSA
}

\begin{abstract}
In this paper, a characterization of Lyapunov graphs associated to smooth flows on surfaces is presented. We first obtain necessary and sufficient conditions for a Lyapunov graph to be associated to Morse-Smale flows and then generalize them to smooth flows. The methods employed in the proofs are of interest in their own right for they introduce the use of the Conley index in this context. Moreover, an algorithmic geometric construction of flows on surfaces is described.
\end{abstract}

\section{INTRODUCTION}

The study of the relationship between flows and topology goes back to Poincare and, in particular, his celebrated theorem which relates the Euler characteristic of a surface $M$ with the global sum of the indices of the singularities of a gradient-flow on $M$.

Later results of Morse [M] generalized Poincaré's result to gradient-like flows on manifolds without boundary. These are now known as the Morse inequalities which relate the index of the singularities of a flow to the dimensions of the homology groups of $M$ (and hence to the Euler characteristic of $M$ ). These inequalities were later generalized by Smale $[\mathrm{S}]$ for Morse-Smale flows and by Zeeman [Z] for Smale flows. In both cases, it was necessary to define an index for the more complex invariant sets.

Smale [S] studied the converse problem of realizing a gradient-like flow on a manifold given a set of nonnegative integers that satisfied the Morse inequalities. This work brought forth the interest in the study of the interplay between the homological properties of a manifold and the dynamics of a flow. More precisely, homological conditions necessary for the existence of a flow are sought and then are shown to be sufficient for a flow to be realized on a manifold.

Conley [C] made a major contribution to this area by generalizing Morse theory. By introducing an index, now known as the Conley index, which generalizes the Morse index he obtained a generalization of the Morse inequalities for smooth flows [CZ].

Conley also proved the existence of Lyapunov functions for smooth flows. A Lyapunov function induces a graph by collapsing components of level sets to distinct points. The vertices of the graph correspond to components of level

Received by the editors October 3, 1991.

1991 Mathematics Subject Classification. Primary 58F25, 58F09, 57R57.

Supported in part by the National Science Foundation under Grant DMS 8704877 and by the Conselho Nacional de Desenvolvimento Científico e Tecnológico under Grant 300072/90.2. 
sets that contain isolated invariant sets and are labelled with the indices of these sets. The edges may be labelled with topological information of the level sets or information on the gluing maps. Such a graph is called a Lyapunov graph and was introduced by Franks in his study of nonsingular Smale flows on the three-sphere [F1].

In this paper, we study Lyapunov graphs of smooth flows on surfaces. The graphs contain the index information (through vertex labelling) as well as homological information of the surface. The theme is to search for conditions on a Lyapunov graph necessary for the existence of a certain class of flows on a given surface. We then proceed to show that these conditions on the graph are sufficient to realize a flow which admits a Lyapunov function with a specified Lyapunov graph.

We use Morse's approach of decomposing a surface $M$ into level sets via a Lyapunov function associated to a flow $\phi_{t}$ on $M$ and then forming the associated Lyapunov graph $\Gamma$. The generalized Morse inequalities are used to determine necessary conditions that $\Gamma$ must satisfy to be associated to $\phi_{t}$. These conditions are shown to be sufficient for the existence of a flow associated to a given abstract Lyapunov graph $\Gamma$ by constructing flows on isolating neighborhoods which correspond to vertices and their incident edges in $\Gamma$. We then glue these isolating neighborhoods together to obtain a flow on $M$ which admits a Lyapunov function realizing the given abstract graph.

We emphasize that the advantage of this characterization over the Morse inequalities is that the Lyapunov graph includes the global disposition of the invariant sets; in particular it contains information about some of their connections. One can view these Lyapunov graphs as a geometric representation of the Morse equality in dimension 2. It is important to note that a Lyapunov graph satisfying the conditions established in this paper does not determine a unique flow up to topological equivalence.

In $\S 2$ we introduce some basic terminology from graph theory and the theory of flows. In $\S 3$, we consider the class of Morse-Smale flows on surfaces, while in $\S 4$ we deal with smooth flows on surfaces.

\section{BACKGROUND}

In this section we introduce the necessary background from graph theory and the theory of flows. A reference for the material on graph theory is $[\mathrm{H}]$.

A digraph (directed graph) is a triple $G=(V, E, \psi)$ where $V$ and $E$ are finite sets and $\psi$ is a map from $E$ to $V \times V$. The elements of $V$ are called vertices and the elements of $E$ are called edges. If $e$ is an edge and $(u, v)=$ $\psi(e)$ we say that $e$ is a directed edge from $u$ to $v$, an outgoing edge of $u$, and an incoming edge of $v$. For the sake of simplifying notation we write $e=(u, v)$ whenever $\psi(e)=(u, v)$. If $v$ is a vertex, then the number of incoming edges of $v$ is called the indegree of $v$ and is denoted $e_{v}^{+}$, the number of outgoing edges of $v$ is called the outdegree of $v$ and is denoted $e_{v}^{-}$, and the sum of the indegree and the outdegree of $v$ is called the degree of $v$ and is denoted $e_{v}$.

Throughout the remainder of the paper we will use the term "graph" to mean a digraph.

A path (between $v_{0}$ and $v_{n}$ ) in a graph is an alternating sequence of vertices and edges, $v_{0}, e_{1}, v_{1}, \ldots, v_{n-1}, e_{n}, v_{n}$ such that for each $i=1, \ldots, n$, ei- 
ther $e_{i}=\left(v_{i}, v_{i-1}\right)$ or $e_{i}=\left(v_{i-1}, v_{i}\right)$. A graph is connected if there is a path between any two vertices in the graph. A path is oriented if for each $i$, $e_{i}=\left(v_{i-1}, v_{i}\right)$. A path is a cycle if the edges are distinct and $v_{0}=v_{n}$. A tree is a connected graph that possesses no cycles. It is not difficult to see that for a tree, the number of vertices less the number of edges is equal to one. The cycle rank of a connected graph $G$ is $\beta=1-V+E$ where $E$ is the number of edges and $V$ the number of vertices. This is sometimes referred to as the (first) Betti number or the cyclomatic number of a connected graph. It follows that the cycle rank of a connected graph is the minimal number of edges whose removal results in a tree.

Two graphs $G_{i}=\left(E_{i}, V_{i}, \psi_{i}\right), i=1,2$, are said to be isomorphic if there is a bijection $\Phi$ between $E_{1}$ and $E_{2}$ and between $V_{1}$ and $V_{2}$ which commutes appropriately with the maps $\psi_{1}$ and $\psi_{2}$.

Let $M$ be a closed surface. In this paper we consider smooth flows $\phi_{t}: M \rightarrow$ $M$ and the isolated invariant sets of the flows. A Lyapunov function $f: M \rightarrow \mathbf{R}$ for $\phi_{t}: M \rightarrow M$ is a function which strictly decreases along orbits of $\phi_{t}$ outside the chain recurrent set and is constant on components of the chain recurrent set. The values of $f$ on the chain recurrent set are called the critical values of $f$. Numbers that are not critical values are called regular values. Combined results of Conley [C] and Wilson [W] establish that any smooth flow admits a smooth Lyapunov function which takes on distinct values on distinct components of the chain recurrent set. We refer to the components of the chain recurrent set as basic sets (cf. [F2]). For the remainder of the paper we assume that $f$ is a smooth Lyapunov function for a smooth flow on the closed surface $M$. If $\Lambda$ is a basic set on which $f$ takes on the critical value $c$, then we say that $\Lambda$ is the level-c basic set. It should be noted that a Lyapunov function for a flow is by no means unique.

If $f$ has finitely many critical values, then it determines a Lyapunov graph. Specifically, a Lyapunov graph $\Gamma$ is obtained from a Lyapunov function $f$ by collapsing to distinct points each component of each level set of $f$. That the resulting space is a graph follows by Proposition 1.4 in [F1]. Each vertex of $\Gamma$ corresponds to a component of $f^{-1}(c)$ containing a basic set. Thus, the value $c$ corresponding to a vertex is a critical value of $f$. The vertices of $\Gamma$ are labelled with information about the corresponding level-c basic set (e.g., the flow on the basic set, the index of the basic set, etc.) The Lyapunov graph is a directed graph, directed by the values of $f$ in the direction of decreasing $f$. As a result it follows that a Lyapunov graph has no oriented cycles (although it may have cycles).

Thus, a Lyapunov function for a flow determines a Lyapunov graph. Conversely, we introduce the notion of an abstract Lyapunov graph and ask when it can be realized as the Lyapunov graph for a Lyapunov function associated to a flow.

An abstract Lyapunov graph is a finite, connected, oriented graph $\Gamma$ which possesses no oriented cycles and each vertex of which is labelled with information about an invariant set [F1].

It is natural to have an equivalence relation among Lyapunov graphs. Let $\Gamma(V, E, \psi)$ and $\Gamma^{\prime}\left(V^{\prime}, E^{\prime}, \psi^{\prime}\right)$ be abstract Lyapunov graphs, we say that $\Gamma$ and $\Gamma^{\prime}$ are equivalent if the underlying graphs are isomorphic via the 
isomorphism $\Phi$ and $v$ is labelled with the same invariant set as $\Phi(v)$. It should be pointed out that the graph does not contain sufficient information to distinguish topological equivalence classes of flows (see the example in Figure 3 below). Given an abstract Lyapunov graph, it can be realized on a closed surface as two nontopologically equivalent flows. The missing information on the graph concerns the relative positions of unstable and stable manifolds.

Results of Peixoto [P] on Morse-Smale flows on closed surfaces distinguish flows up to topological equivalence by making use of a graph whose edges represent the connections of stable and unstable manifolds.

We use the tools of the Conley index theory to establish our results. The Conley index is defined for isolated invariant sets in a flow. A compact subset $N$ of a flow is called an isolating neighborhood if the maximal invariant set in $N$ is contained in the interior of $N$. Under such circumstances the invariant set is said to be isolated.

If $a<b$ are not critical values of $f$, then $f^{-1}([a, b])$ is an isolating neighborhood for the flow. Furthermore, if $\Lambda$ is an isolated level- $c$ basic set, then for $\epsilon>0$, small enough, the component of $f^{-1}([c-\epsilon, c+\epsilon])$ containing $\Lambda$ is an isolating neighborhood for $\Lambda$. We call this isolating neighborhood a basic block for the flow $\phi_{t}$ and the Lyapunov function $f$.(cf. [F]). We denote these basic blocks by $N_{\Lambda, \epsilon}$. Note that $N_{\Lambda, \epsilon}$ is a two-manifold, possibly with boundary. The basic blocks play an important role in defining flows and Lyapunov functions which realize a given abstract Lyapunov graph. By a nonorientable basic set we mean a basic set with a nonorientable basic block $N_{\Lambda, \epsilon}$, and by a nonorientable vertex in a Lyapunov graph we mean a vertex associated to a nonorientable basic set. When no reference is made to orientability, the orientable case is always implied.

Let $N_{\Lambda, \epsilon}$ be a basic block containing the level-c basic set $\Lambda . \Lambda$ corresponds to a vertex $v$ in the associated Lyapunov graph. Consider the sets $N_{\Lambda, \epsilon}^{+}=N_{\Lambda, \epsilon} \cap f^{-1}(c+\epsilon)$ and $N_{\Lambda, \epsilon}^{-}=N_{\Lambda, \epsilon} \cap f^{-1}(c-\epsilon)$. Since $f^{-1}(c+\epsilon)$ and $f^{-1}(c-\epsilon)$ are compact one-manifolds, it follows that $N_{\Lambda, \epsilon}^{+}$and $N_{\Lambda, \epsilon}^{-}$ are unions of circles. It is not difficult to see that each component of $N_{\Lambda, \epsilon}^{+}$ corresponds to an incoming edge of $v$ and that each component of $N_{\Lambda, \epsilon}^{-}$corresponds to an outgoing edge of $v$.

The index of an isolated invariant set $\Lambda$ is defined in terms of index pairs $\left(N_{1}, N_{0}\right)$, where $N_{1}$ is an isolating neighborhood of $\Lambda$ and $N_{0}$ acts as an exit set for $N_{1}$ (see [C]). For example, if $\Lambda$ is a level- $c$ basic set, then $\left(N_{\Lambda, \epsilon}, N_{\Lambda, \epsilon}^{-}\right)$ is an index pair for $\Lambda$. We call such an index pair a block-defined index pair.

The choice of an index pair $\left(N_{1}, N_{0}\right)$ for an isolated invariant set $\Lambda$ is not unique; however, Conley [C] proves that the homotopy type of the pointed quotient space $N_{1} / N_{0}$ is independent of the pair. The resulting homotopy type of the quotient space is called the Conley index of $\Lambda$ and is denoted $h(\Lambda)$. Define $C H_{*}(\Lambda)$, the Conley homology index of $\Lambda$, to be the homology of the Conley index of $\Lambda$; i.e. $C H_{*}(\Lambda)=H_{*}(h(\Lambda))$.

If $\left(N_{1}, N_{0}\right)$ is an index pair for $\Lambda$, then the Conley homology index of $\Lambda$ is equal to $H_{*}\left(N_{1} / N_{0}\right)$, the homology of the pointed quotient space $N_{1} / N_{0}$. This need not be isomorphic to $H_{*}\left(N_{1}, N_{0}\right)$, the homology of the pair of spaces $\left(N_{1}, N_{0}\right)$. However, if $\left(N_{1}, N_{0}\right)$ is a block-defined index pair, then 
since $f^{-1}(c-\epsilon)$ is a deformation retract of a neighborhood of itself in $f^{-1}([c-\epsilon, c+\epsilon])$, it follows that $N_{0}$ is a deformation retract of a neighborhood of itself in $N_{1}$, and therefore, by standard algebraic topology results (see [S]), $H_{*}\left(N_{1}, N_{0}\right)$ is isomorphic to $H_{*}\left(N_{1} / N_{0}\right)$ and therefore to $C H_{*}(\Lambda)$.

\section{Morse-SMale flows on SURfaces}

A smooth flow $\phi_{t}$ on $M$ is a Morse-Smale flow if the chain recurrent set consists of hyperbolic singularities and closed orbits and the unstable manifold of any singularity or closed orbit has transversal intersection with the stable manifold of any singularity or closed orbit. Each basic set for a Morse-Smale flow is either a singularity or a periodic orbit. Each singularity is either attracting (a sink), repelling (a source), or a saddle, and each periodic orbit is either attracting or repelling.

Below, we classify the basic blocks $N_{\Lambda, \epsilon}$ associated to the basic sets under consideration. For attracting and repelling singularities, such a basic block must be a disk. For saddles and periodic orbits, such a basic block might be either orientable or nonorientable.

Since the basic sets under consideration in this section are only of the types listed above (attracting and repelling singularities, attracting and repelling periodic orbits that are orientable or nonorientable, and saddles that are orientable or nonorientable), we label the vertices on (abstract) Lyapunov graphs accordingly.

The following theorem gives necessary and sufficient conditions for an abstract Lyapunov graph to be associated with a Morse-Smale flow and a Lyapunov function on a closed surface.

Theorem 1. Let $M$ be a closed surface. An abstract Lyapunov graph $\Gamma$ is associated to a Morse-Smale flow $\phi_{t}$ and a Lyapunov function $f$ on $M$ if and only if the following conditions are satisfied:

(1) (local conditions) If the vertex $v$ is labelled with $\Lambda$ and

(a) $\Lambda$ is a source (sink) then the number of exiting (entering) edges $e_{v}^{-}\left(e_{v}^{+}\right)$is equal to one.

(b) $\Lambda$ is a saddle then $1 \leq e_{v}^{+} \leq 2,1 \leq e_{v}^{-} \leq 2$ and $e_{v}^{+}+e_{v}^{-} \leq 3$. Moreover $\Lambda$ is orientable if and only if $e_{v}^{+}+e_{v}^{-}=3$.

(c) $\Lambda$ is a repelling (attracting) periodic orbit then $e_{v}^{-} \leq 2\left(e_{v}^{+} \leq 2\right)$. Moreover $\Lambda$ is orientable if and only if $e_{v}^{-}=2\left(e_{v}^{+}=2\right)$.

(2) (global conditions)

(a) If $M$ is orientable, the cycle rank of $\Gamma$ must be equal to the genus of $M$.

(b) If $M$ is nonorientable, twice the cycle rank of $\Gamma$ plus the number of nonorientable vertices must be equal to the genus of $M$.

Intuitively the global conditions imply that each cycle of the graph (in the sense of a one-complex) corresponds to the addition of a handle $S^{1} \times D^{1}$ to $S^{2}$ in the orientable case. Hence each handle increases the genus of the manifold by one. In the nonorientable case, the cycles represent the addition of a handle to 
$R P^{2}$. However, in this case, each handle increases the genus by two. And each nonorientable vertex corresponds to the addition of a Möbius band to $R P^{2}$, which increases the genus by one.

Proof (Necessity of the local conditions). Let $\Gamma$ be the Lyapunov graph associated with a Morse-Smale flow $\phi_{t}$ and a Lyapunov function $f$ on the closed surface $M$. In studying the basic sets, we use the Conley homology index and compute with coefficients in $\mathbf{Z}_{2}$. Let $\Lambda$ be a basic set. Note that $C H_{n}(\Lambda)=0$ if $n>2$, otherwise homology indices are as follows:

\begin{tabular}{|l|c|c|c|}
\hline TYPE OF BASIC SET $\Lambda$ & $\mathrm{CH}_{2}(\Lambda)$ & $\mathrm{CH}_{1}(\Lambda)$ & $C H_{0}(\Lambda)$ \\
\hline Attracting Singularity & 0 & 0 & $\mathbf{Z}_{2}$ \\
\hline Repelling Singularity & $\mathbf{Z}_{2}$ & 0 & 0 \\
\hline Saddle & 0 & $\mathbf{Z}_{2}$ & 0 \\
\hline Attracting Periodic Orbit & 0 & $\mathbf{Z}_{2}$ & $\mathbf{Z}_{2}$ \\
\hline Repelling Periodic Orbit & $\mathbf{Z}_{2}$ & $\mathbf{Z}_{2}$ & 0 \\
\hline
\end{tabular}

Now assume that $\left(N_{1}, N_{0}\right)$ is a block-defined index pair for $\Lambda$. Since $\Lambda$ is not all of $M$, it follows that $N_{1}$ has boundary. Since $N_{1}$ is a connected two-manifold with boundary, it follows that $H_{2}\left(N_{1}\right)=\widetilde{H}_{0}\left(N_{1}\right)=0$ (where $\widetilde{H}_{*}$ denotes reduced homology). If $N_{0}$ is nonempty, then since $N_{0}$ is a closed one-manifold, it follows that $N_{0}$ is a union of circles. For such $N_{0}$ we have the following long exact homology sequence:

$$
0 \rightarrow C H_{2}(\Lambda) \stackrel{\partial_{2}}{\rightarrow} H_{1}\left(N_{0}\right) \stackrel{i_{1}}{\rightarrow} H_{1}\left(N_{1}\right) \stackrel{p_{1}}{\rightarrow} C H_{1}(\Lambda) \stackrel{\partial_{1}}{\rightarrow} \widetilde{H}_{0}\left(N_{0}\right) \rightarrow 0
$$

Let $v$ be the vertex of $\Gamma$ labelled with $\Lambda$. It easily follows that $\operatorname{dim} H_{0}\left(N_{0}\right)=$ $e_{v}^{-}$, and therefore if $N_{0}$ is nonempty, then $\operatorname{dim} \widetilde{H}_{0}\left(N_{0}\right)=e_{v}^{-}-1$. Since $N_{0}$ is a union of circles, we also have $\operatorname{dim} H_{1}\left(N_{0}\right)=e_{v}^{-}$.

Assume $\Lambda$ is a repelling singularity. Clearly $e_{v}^{+}=0$ and $N_{0}$ is nonempty. Using the homology sequence, $\operatorname{dim} C H_{1}(\Lambda)=0$ implies that $\operatorname{dim} \widetilde{H}_{0}\left(N_{0}\right)=0$ and therefore $e_{v}^{-}=1$. By reversing the flow, it similarly follows that if $v$ is labelled with an attracting singularity, then $e_{v}^{+}=1$ and $e_{v}^{-}=0$. Thus local condition (a) follows.

Now assume $\Lambda$ is a saddle. Clearly $N_{0}$ is nonempty and $e_{v}^{-} \geq 1$. Using the homology sequence, $\operatorname{dim} C H_{1}(\Lambda)=1$ implies that $\operatorname{dim} \widetilde{H}_{0}\left(N_{0}\right) \leq 1$ and therefore $e_{v}^{-} \leq 2$. By reversing the flow it similarly follows that $1 \leq e_{v}^{+} \leq 2$.

To complete the proof of the local conditions for a saddle vertex, we identify the possible basic blocks, $N_{1}$, of the saddle $\Lambda$, up to homeomorphism. From this classification it is shown that $e_{v}^{+}+e_{v}^{-} \leq 3$ with strict inequality holding if and only if $N_{1}$, and therefore $v$, is nonorientable.

We begin by showing that $\operatorname{dim} H_{1}\left(N_{1}\right)=2$. There are two cases to consider. First if $e_{v}^{-}=1$, then $\operatorname{dim} \widetilde{H}_{0}\left(N_{0}\right)=0$, and the homology sequence is short exact. From this $\operatorname{dim} H_{1}\left(N_{1}\right)=2$ easily follows. Second if $e_{v}^{-}=2$ then $\partial_{1}$ is an isomorphism, which, with $\operatorname{dim} \mathrm{CH}_{2}(\Lambda)=0$ implies $H_{1}\left(N_{0}\right) \approx H_{1}\left(N_{1}\right)$. Since $\operatorname{dim} H_{1}\left(N_{0}\right)=e_{v}^{-}=2$, it then follows that $\operatorname{dim} H_{1}\left(N_{1}\right)=2$. 
Now, $N_{1}$ is a connected two-manifold with nonempty boundary. Since $\operatorname{dim} H_{1}\left(N_{1}\right)=2$, it follows thiat $\chi\left(N_{1}\right)$, the Euler characteristic of $N_{1}$, equals -1 . Let $N_{1}$ have $b$ boundary components and genus $g$; then if $N_{1}$ is orientable, then $2-2 g-b=\chi\left(N_{1}\right)=-1$, and if $N_{1}$ is nonorientable, then $2-g-b=\chi\left(N_{1}\right)=-1$. Consider the various possibilities for $e_{v}^{-}$and $e_{v}^{+}$, and therefore for $b$. If $e_{v}^{-}=e_{v}^{+}=1$, then $b=2$. This can only occur if $N_{1}$ is nonorientable and has genus 1 ( $N_{1}$ would be a Möbius band with a hole). If $e_{v}^{+}+e_{v}^{-}=3$, then $b=3$, and similar to above, it can easily be seen that $N_{1}$ must be a disk with two holes. Finally, if $e_{v}^{-}=e_{v}^{+}=2$, then $b=4$. This yields $g \leq 0$, which is not possible. Thus $e_{v}^{+}+e_{v}^{-} \leq 3$ with equality holding if and only if $\Lambda$ is orientable.

Now assume $\Lambda$ is a repelling periodic orbit. Clearly $e_{v}^{+}=0$ and $\operatorname{dim} C H_{1}(\Lambda)=\operatorname{dim} C H_{2}(\Lambda)=1$. By the surjectivity of $\partial_{1}$, it follows that $e_{v}^{-} \leq 2$. As above, to complete the proof of the local conditions for a repelling periodic orbit vertex, we identify the possible basic blocks $N_{1}$. By separating into the cases $e_{v}^{-}=1$ or $e_{v}^{-}=2$, it is not difficult to see from the homology sequence that $\operatorname{dim} H_{1}\left(N_{1}\right)=1$. Now, $N_{1}$ is a connected two-manifold with nonempty boundary. Since $\operatorname{dim} H_{1}\left(N_{1}\right)=1$, it follows that $\chi\left(N_{1}\right)=0$. Similar to above, it follows that if $e_{v}^{-}=1$, then $N_{1}$ is a Möbius band, and if $e_{v}^{-}=2$, then $N_{1}$ is an annulus.

It now follows that if $\Lambda$ is a repelling periodic orbit, then $e_{v}^{+}=0$ and $e_{v}^{+} \leq 2$, with equality holding if and only if $v$ is orientable. The corresponding result for attracting periodic orbits follows by reversing the flow. The proof of the necessity of the local conditions is now complete.

It is noteworthy that all the possible basic blocks, $N_{1}$, described in the above proof are realizable. This, of course, plays an important role in the proof of the sufficiency of the conditions in Theorem 1. The various possibilities are illustrated in Figure 1 below. The basic blocks are glued together appropriately to define a flow on $M$ that realizes a given abstract Lyapunov graph, thereby establishing the sufficiency of the global condition. First, we prove the necessity of the global conditions in Theorem 1 and then we will establish the sufficiency result.

Lemma 1 is used in the proof of the necessity of the global conditions in Theorem 1. Lemma 1 relates the homology index of the basic set and the number of boundary components in the basic block to the genus of the basic block. The latter, in the case of a Morse-Smale flow, is either zero (if the basic block is orientable) or one (if the basic block is not orientable). The proof follows easily by considering the various cases for basic blocks in Figure 1.

Lemma 1. Let $N$ be a basic block for a Morse-Smale flow on a closed surface. Assume that $N$ has $b$ boundary components. Let $h_{j}$ denote the dimension of the jth Conley homology index of the basic set isolated by $N$. Then $-h_{2}+h_{1}-$ $h_{0}-b+2$ equals zero if $N$ is orientable and equals one if $N$ is nonorientable.

Proof (Necessity of the global conditions). Let $\Gamma$ be a Lyapunov graph associated with a Morse-Smale flow $\phi_{t}$ and a Lyapunov function $f$ on a closed surface $M$. Assume that $\Gamma$ has $V$ vertices, $E$ edges and cycle rank $\beta$. 

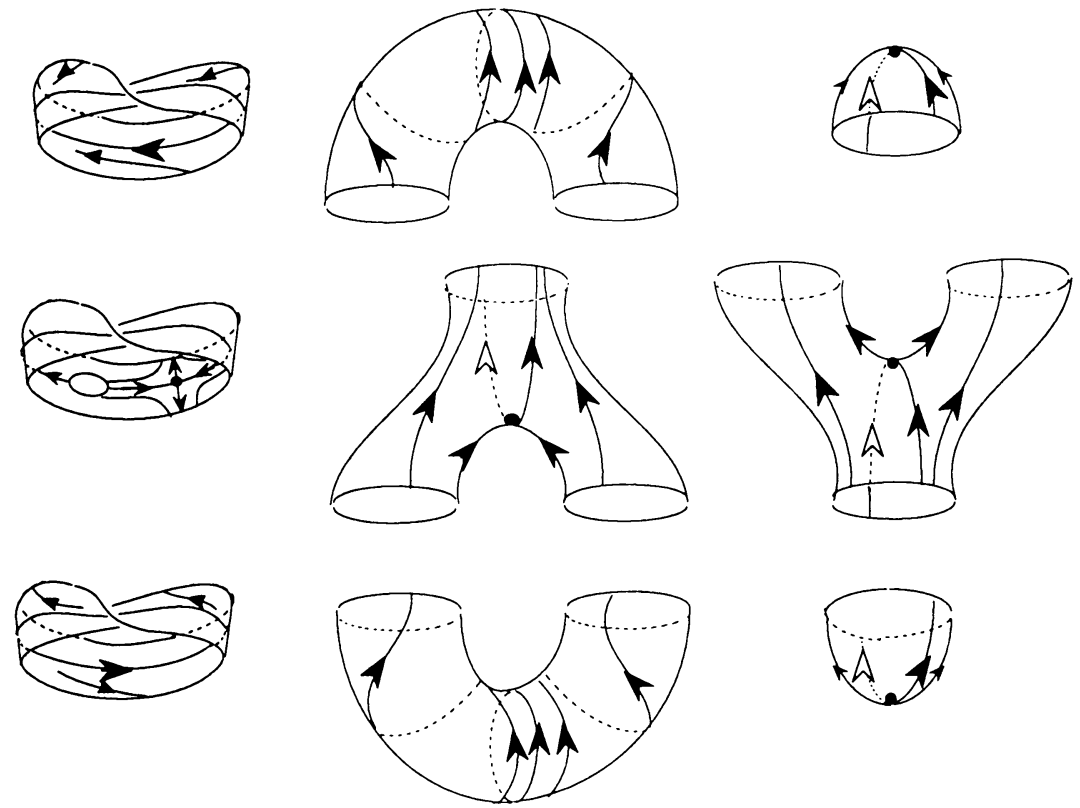

FIGURE 1. Basic blocks for Morse-Smale flows on surfaces

The necessity of the global conditions is based on the Morse equality [CZ], which states that

$$
\chi(M)=\sum_{i=1}^{V}\left(h_{2}^{i}-h_{1}^{i}+h_{0}^{i}\right)
$$

where $\chi(M)$ is the Euler characteristic of $M$ and $h_{j}^{i}$ is the dimension of the $j$ th Conley homology index of the $i$ th basic set and the sum is taken over all the basic sets.

Let $b_{i}$ be the number of boundary components in a basic block associated to the $i$ th basic set. Then the corresponding vertex on the Lyapunov graph must have degree $b_{i}$, and therefore $E$, the total number of edges in the graph, is equal to $\sum_{i=1}^{V} \frac{b_{i}}{2}$. By definition, $V-E+\beta=1$, from which it follows that

$$
V-\sum_{i=1}^{V} \frac{b_{i}}{2}+\beta=1 .
$$

Assume that $M$ is orientable of genus $G$. Then

$$
\begin{aligned}
G & =\frac{2-\chi(M)}{2}=1-\sum_{i=1}^{V} \frac{\left(h_{2}^{i}-h_{1}^{i}+h_{0}^{i}\right)}{2} \\
& =\beta+\sum_{i=1}^{V}\left(-\frac{b_{i}}{2}+1\right)-\sum_{i=1}^{V} \frac{\left(h_{2}^{i}-h_{1}^{i}+h_{0}^{i}\right)}{2} \\
& =\beta+\sum_{i=1}^{V} \frac{-h_{2}^{i}+h_{1}^{i}-h_{0}^{i}-b_{i}+2}{2}=\beta
\end{aligned}
$$


where the second equality holds by the Morse equality, the third by equation 1 , and the last, using the fact that every vertex must be orientable, by Lemma 1 . Thus, the necessity of the global conditions in Theorem 1 holds in the case that $M$ is orientable.

Now assume that $M$ is nonorientable of genus $G$ and the Lyapunov graph has $\mu$ nonorientable vertices. Then, as above

$$
\begin{aligned}
G & =2-\chi(M)=2-\sum_{i=1}^{V}\left(h_{2}^{i}-h_{1}^{i}+h_{0}^{i}\right) \\
& =2 \beta+\sum_{i=1}^{V}\left(-b_{i}+2\right)-\sum_{i=1}^{V}\left(h_{2}^{i}-h_{1}^{i}+h_{0}^{i}\right) \\
& =2 \beta+\sum_{i=1}^{V}-h_{2}^{i}+h_{1}^{i}-h_{0}^{i}-b_{i}+2=2 \beta+\mu
\end{aligned}
$$

It follows that the global conditions hold for $M$ nonorientable, and the proof is complete.

We have shown that given a Morse-Smale flow on $M$, its Lyapunov graph satisfies the local and global conditions given in (1) and (2) of Theorem 1. Next we will prove the converse, that is, given an abstract Lyapunov graph $\Gamma$ satisfying (1) and (2), a Morse Smale flow can be realized on $M$ with Lyapunov graph equivalent to $\Gamma$, thus concluding the proof of Theorem 1 .

Proof (Sufficiency of the local and global conditions). We must prove that given an abstract Lyapunov graph satisfying the local and global conditions as described in the statement of the theorem, then there is a Morse-Smale flow on $M$ with Lyapunov function whose associated Lyapunov graph is as given.

This is done by appropriately gluing together basic blocks, so that, first of all, we obtain a smooth flow and secondly, one in which stable and unstable manifolds of the basic sets intersect transversally.

Begin by numbering the vertices in the graph, 1, 2, 3, etc., such that the resulting ordering respects the ordering in the graph. Consider vertex $j$. Depending on the labelling of the vertex and on the number of incoming and outgoing edges to that vertex, there is a basic block from Figure 1 associated to it. We may assume that the basic block is of the form $f^{-1}\left(\left[j-\frac{1}{2}, j+\frac{1}{2}\right]\right)$ for a function $f$ that serves as a Lyapunov function for the resulting flow. The desired flow on $M$ is obtained by gluing these basic blocks together as follows. Assume vertices $j$ and $k$, where $j+1 \leq k$, are connected by an edge.Then the basic block for vertex $j$ has a boundary component in $f^{-1}\left(j+\frac{1}{2}\right)$ and the flow is entering through this component, while the basic block for vertex $k$ has a boundary component in $f^{-1}\left(k-\frac{1}{2}\right)$ and the flow is exiting through this component. If $k=j+1$ then glue the basic blocks together along these boundary components. If $k>j+1$ then glue these basic blocks appropriately to a cylinder on which there is a longitudinal flow with a Lyapunov function $f$ which takes on the value $k-\frac{1}{2}$ on the entering boundary component and $\left(j+\frac{1}{2}\right)$ on the exiting boundary component (see Figure 2). Note, if there are 

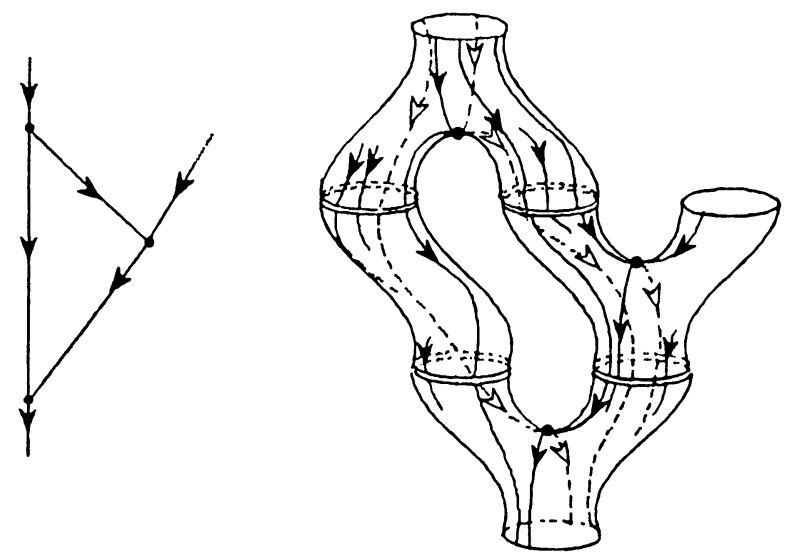

FIGURE 2. Gluing basic blocks according to a Lyapunov graph

two such edges connecting vertex $j$ and vertex $k$, then there are two pairs of boundary components to be attached.

Furthermore, note that some care must be taken in performing the gluing so that the resulting flow is Morse-Smale and so that the resulting manifold is homeomorphic to $M$; more will be said about this below.

It is clear that if the above gluing is appropriately done, then the result of the construction is a closed surface on which there is defined a smooth flow with Lyapunov function $f$ having Lyapunov graph as given.

To insure that the flow is Morse-Smale, it is necessary to glue the basic blocks together so that the stable and unstable manifolds of the basic sets intersect transversally. For the two-dimensional case under consideration here, it is only necessary to glue the basic blocks so that no saddle connections occur.

We can assume without loss of generality, that the above construction starts with a saddle basic block and glues on all saddle basic blocks before gluing on other types of basic blocks. Note that the unstable (stable) manifold of a saddle partitions the exiting (entering) boundary components of the saddle basic block into two disjoint arcs. Similarly, a union of saddle basic blocks $S^{j}=\bigcup_{i=1}^{j} S_{i}$ has its exiting (entering) set partitioned by the unstable (stable) manifolds of some of the saddles in $S^{j}$. To insure that no saddle connections occurred in $S^{j}$ we must verify that all of the unstable manifolds of the $j$ saddles in $S^{j}$ intersect with the exiting set of $S^{j}$. In other words, the exiting set of $S^{j}$ must be partitioned into $2 j$ disjoint arcs (see Figure 2). If this condition is verified for $S^{J}$ where $J$ is the total number of saddles then the flow constructed thus far satisfies the transversality condition.

Note that there may be a number of ways to perform this gluing resulting in qualitatively distinct flows (see Figure 3), and thus the Lyapunov graph fails as a complete classifier of Morse-Smale flows on surfaces. If one were to codify the possible intersections that occur between all stable and unstable manifolds as the basic sets are glued together and add that information to the information in the Lyapunov graph, then the result would be a complete classifier of the Morse-Smale flows on surfaces. That is roughly how Peixoto's classification scheme works $[\mathrm{P}]$, although it is done with a graph that is different from the Lyapunov graph. We do not pursue that fine a classification here. 


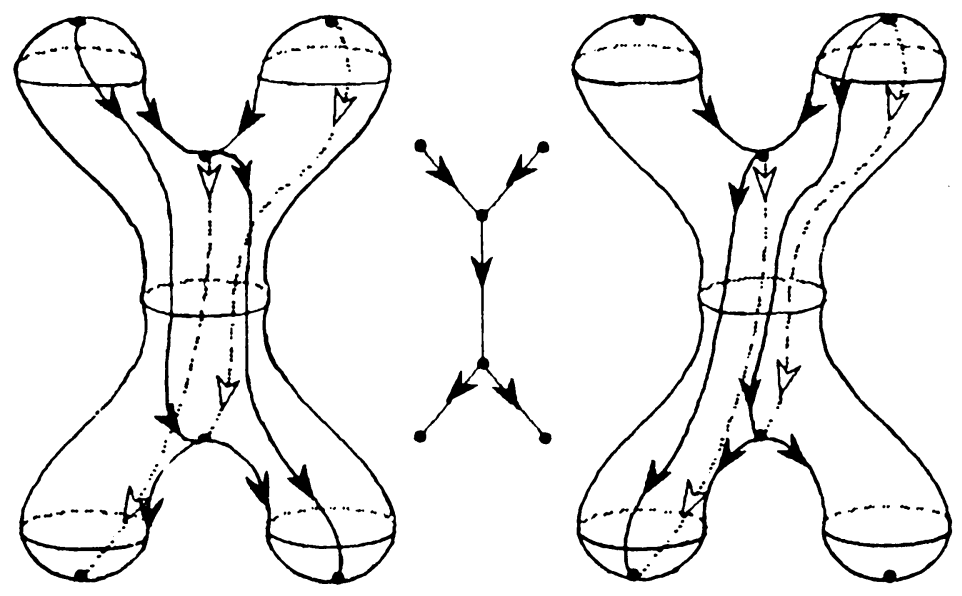

Figure 3. Qualitatively distinct flows resulting from the same abstract Lyapunov graph, and arising from different gluings along the middle boundary component of the basic blocks

Under certain circumstances it is necessary to take care when gluing the basic blocks together so that the resulting manifold is (homeomorphic to) M. Note that since the Lyapunov graph of the resulting Lyapunov function $f$ is the original abstract Lyapunov graph, and since the graph satisfies the global conditions in the statement of the theorem, it follows that the genus of the resulting manifold equals that of $M$. The question is only one of orientation, and if the resulting manifold and $M$ are either both orientable or both nonorientable, then they are homeomorphic. Now, if $M$ is orientable, then all of the basic blocks are orientable, and if they are glued together preserving orientation, then the resulting manifold is orientable and thus homeomorphic to $M$. If $M$ is nonorientable and there is a nonorientable basic block, then the resulting manifold is nonorientable and thus homeomorphic to $M$. Finally, if $M$ is nonorientable and there are no nonorientable basic blocks, then (as the reader can verify) if the resulting manifold is constructed by gluing up from the bottom (as described in the previous paragraph), then at some point in the construction it is necessary to glue a basic block for an orientable saddle with $e^{-}=2$ and $e^{+}=1$ (see Figure 1) onto a connected orientable manifold. That gluing can be done so that orientation is not preserved (by "twisting" one of the two exiting boundary components on the basic block), and thus the resulting manifold is nonorientable and homeomorphic to $M$.

Corollary 1. If $\Gamma$ is the Lyapunov graph associated to a Morse-Smale flow and a Lyapunov function on a closed surface, then $\Gamma$ is planar.

The necessity of $\Gamma$ being planar is specific to the setting under consideration because the necessity is lost when the Morse-Smale condition is dropped. The converse of the corollary does not hold in general; i.e. $\Gamma$ planar does not necessarily imply that $\phi_{t}$ is Morse-Smale.

We will now prove Corollary 1. 
Proof. This follows easily from a result due to Kuratowski which asserts that any nonplanar graph must contain a subgraph which is either $K_{5}$ or $K_{3,3}$. Since the degree of $\Gamma$ must be at most three, $K_{5}$ is not realizable nor is any graph containing $K_{3,3} . K_{3,3}$ is also not realizable as a Morse-Smale flow, since any orientation on $K_{3,3}$ will either have oriented cycles or a vertex of indegree or outdegree equal to three, which is not possible for Lyapunov graphs on closed surfaces.

\section{SMOOTH FLOWS ON SURFACES}

Let $M$ be a closed surface. In this section we consider smooth flows whose chain recurrent set has finitely many components. Such a chain recurrent set we call a finite-component chain recurrent set. For example, if the chain recurrent set is hyperbolic, then since $M$ is compact it follows that the chain recurrent set is a finite-component chain recurrent set [S]. However, there are examples of a finite-component chain recurrent set which contains infinitely many singularities or closed orbits. For example, consider the flow on the two-sphere with a twocomponent chain recurrent set: the north pole being a repelling singularity, orbits in the northern hemisphere spiraling down towards the equator and the southern hemisphere consisting of infinitely many periodic orbits with the south pole being a center.

On the other hand, there are examples where the chain recurrent set has infinitely many components. For instance, consider on the two-sphere the flow which has an attracting singularity at the south pole and alternating repelling and attracting periodic orbits (parallel to the equator) $P_{1 / n}$ at distances $\frac{1}{n}$ from the north pole for $n \geq 1$. The theory that we present here can easily be generalized to the case where the chain recurrent set has infinitely many components. In this setting one considers Morse decompositions [C], a decomposition of $M$ into finitely many sets called Morse sets each of which might contain many components of the chain recurrent set along with orbits connecting these components. However, we will not consider that generalization in this paper.

Let $\Gamma$ be an abstract Lyapunov graph associated to a smooth flow on $M$, and let $v$ be a vertex of $\Gamma$ labelled with Conley homology indices with dimension $h_{j}$ for $j=0,1,2$. The genus of $v, g_{v}$ is defined by

$$
g_{v}= \begin{cases}\frac{-h_{2}+h_{1}-h_{0}-e_{v}+2}{2} & \text { if } M \text { is orientable; } \\ \left(-h_{2}+h_{1}-h_{0}-e_{v}+2\right) & \text { if } M \text { is nonorientable. }\end{cases}
$$

If $N$ is a basic block for the basic set corresponding to vertex $v$ in the above definition, then if $M$ is orientable or $N$ is nonorientable, then $g_{v}$ is the genus of the two-manifold $N$. In any case, in the following theorem we see that $g_{v}$ is the contribution from $N$ to the genus of the manifold $M$.

Theorem 2. Let $M$ be a closed surface and let $\Gamma$ be an abstract Lyapunov graph whose vertices are labelled with homology indices of dimensions $h_{0}, h_{1}$ and $h_{2}$. Then $\Gamma$ is associated to a smooth flow on $M$ with a finite-component chain recurrent set and a Lyapunov function $f$ if and only if the following conditions are satisfied: 
(1) (local conditions) If $v$ is a vertex labelled with Conley homology indices of dimensions $h_{j}$ for $j=0,1,2$ then

$$
e_{v}^{+} \leq h_{1}+1, \quad e_{v}^{-} \leq h_{1}+1, \quad e_{v}^{+}+e_{v}^{-} \leq h_{1}-h_{2}-h_{0}+2 .
$$

where the latter inequality is strict if $v$ is nonorientable. Furthermore, if $e_{v}^{+}=0$ then $h_{2}=1$, otherwise $h_{2}=0$; if $e_{v}^{-}=0$ then $h_{0}=1$, otherwise $h_{0}=0$.

(2) (global conditions) The genus of each vertex is a nonnegative integer, and if $M$ has genus $G$ then

$$
G= \begin{cases}\beta+\sum_{i=1}^{V} g_{v_{i}} & \text { if } M \text { is orientable; } \\ 2 \beta+\sum_{i=1}^{V} g_{v_{i}} & \text { if } M \text { is nonorientable. }\end{cases}
$$

where the sums are taken over all the $V$ vertices in $\Gamma$.

The global conditions in Theorem 1 can be seen to easily follow from the global conditions here. In particular, Lemma 1 implies that $g_{v}=0$ if $v$ is orientable and $g_{v}=1$ if $v$ is nonorientable. Thus in Theorem 1 if $M$ is orientable then $G=\beta$, and if $M$ is non-orientable then $G=2 \beta+$ (the number of nonorientable vertices).

The global conditions in Theorem 2 give a precise breakdown of $M$ in terms of contributions from the graph and contributions from the basic blocks. For example, if $M$ is orientable, then $M$ is a connected sum of tori, and the connected sum gets a contribution of one torus from each cycle in the graph $\Gamma$ and a contribution of $g_{v}$ tori from each vertex $v$ in the graph (and therefore from each of the corresponding basic blocks). The breakdown is similar for $M$ nonorientable.

Proof (Necessity of local and global conditions). If $\Gamma$ consists of a single vertex then the flow on $M$ is recurrent. $M$ with the recurrent flow is the basic set corresponding to the vertex, and the Conley homology index of this invariant set equals the homology of the manifold $M$. It is not difficult to see that the desired inequalities and equations hold.

Now assume that $\Gamma$ has more than one vertex, and let $\Lambda$ be a level- $c$ basic set for the Lyapunov function $f$. Recall that $h_{2}=\operatorname{dim} C H_{2}(\Lambda), h_{1}=$ $\operatorname{dim} C H_{1}(\Lambda)$, and $h_{0}=\operatorname{dim} C H_{0}(\Lambda)$.

Assume that $\left(N_{1}, N_{0}\right)$ is a block-defined index pair for $\Lambda$ and let $v$ be the vertex of $\Gamma$ associated to $\Lambda$. Since $\Lambda$ is not all of $M$ (because $\Gamma$ contains more than one vertex), it follows that $N_{1}$ has boundary. Since $N_{1}$ is a connected two-manifold with boundary, it follows that $H_{2}\left(N_{1}\right)=\widetilde{H}_{0}\left(N_{1}\right)=0$ (where $\widetilde{H}_{*}$ denotes reduced homology). It can be easily seen that $\operatorname{dim} H_{0}\left(N_{0}\right)=e_{v}^{-}$and thus if $N_{0}$ is empty, then $e_{v}^{-} \leq h_{1}+1$ clearly holds. If $N_{0}$ is nonempty, then since $N_{0}$ is a closed one-manifold, it follows that $N_{0}$ is a union of circles. For such $N_{0}$ we have the following long exact homology sequence:

$$
0 \rightarrow C H_{2}(\Lambda) \rightarrow H_{1}\left(N_{0}\right) \rightarrow H_{1}\left(N_{1}\right) \rightarrow C H_{1}(\Lambda) \stackrel{\partial_{1}}{\rightarrow} \widetilde{H}_{0}\left(N_{0}\right) \rightarrow 0
$$

Since $N_{0}$ is nonempty, it follows that $\operatorname{dim} \widetilde{H}_{0}\left(N_{0}\right)=e_{v}^{-}-1$. Since $N_{0}$ is a union of circles, we also have $\operatorname{dim} H_{1}\left(N_{0}\right)=e_{v}^{-}$. By exactness, $\operatorname{dim}\left(\operatorname{Im} \partial_{1}\right)=$ $e_{v}^{-}-1$ and $\operatorname{dim}\left(\operatorname{ker} \partial_{1}\right)=h_{1}-\left(e_{v}^{-}-1\right) \geq 0$. It follows that $e_{v}^{-} \leq h_{1}+1$. 
Now, if $\Lambda$ is a repeller, then $e_{v}^{+}=0$ and therefore $e_{v}^{+} \leq h_{1}+1$. Assume $\Lambda$ is not a repeller. Reversing the flow clearly interchanges $e_{v}^{+}$and $e_{v}^{-}$. If $\Lambda^{\prime}$ is thie corresponding basic set in the reversed flow then (as the reader can verify) if $\Lambda$ is not an attractor, then $C H_{1}\left(\Lambda^{\prime}\right)=C H_{1}(\Lambda)$, and if $\Lambda$ is an attractor, then $C H_{1}\left(\Lambda^{\prime}\right)=C H_{1}(\Lambda)-1$. In either case if we apply the inequality $e_{v}^{-} \leq h_{1}+1$ to the reversed flow and make the appropriate substitutions, it follows that $e_{v}^{+} \leq h_{1}+1$.

To obtain the upper bounds for $e_{v}^{+}+e_{v}^{-}$, we use the fact that the Euler characteristic for an orientable $N_{1}$ is $\chi\left(N_{1}\right)=2-2 g-b$ where $g$ is the genus and $b=e_{v}^{+}+e_{v}^{-}$is the number of boundary components. On the other hand, $\chi\left(N_{1}\right)=h_{2}-h_{1}+h_{0}$. Hence, $2 g=h_{1}-h_{0}-h_{2}-b+2 \geq 0$ and consequently, $e_{v}^{+}+e_{v}^{-} \leq h_{1}-h_{2}-h_{0}+2$. If $N_{1}$ is nonorientable then $\chi\left(N_{1}\right)=2-g-b$ and $g=h_{1}-h_{0}-h_{2}-b+2>0$ proving the inequality $e_{v}^{+}+e_{v}^{-}<h_{1}-h_{2}-h_{0}+2$.

Now if $e_{v}^{+}=0$, then $N_{0}=\partial N_{1}$, from which it follows that $H_{2}\left(N_{1}, N_{0}\right)=$ $\mathbf{Z}_{2}$. If $e_{v}^{+} \neq 0$, then there are components of $\partial N_{1}$ that are disjoint from $N_{0}$, implying that $H_{2}\left(N_{1}, N_{0}\right)=0$. Thus if $e_{v}^{+}=0$ then $h_{2}=1$, otherwise $h_{2}=0$.

If $e_{v}^{-}=0$, then $N_{0}$ is empty, and since $N_{1}$ is connected, it follows that $H_{0}\left(N_{1}, N_{0}\right)=\mathbf{Z}_{2}$. If $e_{v}^{-} \neq 0$ then $N_{0}$ is nonempty, and since $N_{1}$ is connected, it follows that $H_{0}\left(N_{1}, N_{0}\right)=0$. Thus if $e_{v}^{-}=0$ then $h_{0}=1$, otherwise $h_{0}=0$.

It can be easily verified that the genus of each vertex is a nonnegative integer.

The proof of the necessity of the global conditions follows along the lines of the corresponding proof in Theorem 1. It is not difficult to see that if the $g_{v}$, the genera of the vertices, are substituted appropriately in equation (2) and equation (3), then the global conditions in the statement of Theorem 2 follow.

To prove the sufficiency result in Theorem 1, basic blocks are glued together to provide a Morse-Smale flow on $M$ that has a Lyapunov function $f$ such that the resulting Lyapunov graph is as given. The flow on the basic block is particularly simple, and only these simple pieces are needed to establish the result. To establish the general sufficiency result in Theorem 2 above, we similarly glue basic blocks together to obtain a flow with a Lyapunov function such that a particular Lyapunov graph is realized. The following theorem indicates that any compact two-manifold with separate entering and/or exiting boundary components acts as a basic block for a flow with a particularly simple basic set (a singularity if there are both entering and exiting boundary components, a singularity with homoclinic orbits if there are either only entering or only exiting boundary components). This theorem is used in the proof of the sufficiency result in Theorem 2; it guarantees the existence of the basic blocks that are needed to build the desired flow on $M$. We discuss the proof of the sufficiency of the local and global conditions in Theorem 2 following the proof of Theorem 3.

Theorem 3. Let $N$ be a genus $g$ two-manifold with boundary. Label $e^{+}$boundary components of $N$ as entering and $e^{-}$as exiting. Then $N$ is a basic block for a flow which respects the boundary labelling (i.e. the flow enters through the entering boundary components and exits through the exiting boundary components), and is such that the basic set in $N$ is: 
(1) A singularity with $h_{1}=2 g-2+e^{+}+e^{-}$if $e^{+}, e^{-}>0$ and $N$ is orientable.

(2) A singularity with $h_{1}=g-2+e^{+}+e^{-}$if $e^{+}, e^{-}>0$ and $N$ is nonorientable.

(3) A singularity with $2 g+e^{+}+e^{-}-1$ homoclinic orbits if either $e^{+}=0$ or $e^{-}=0$, but not both, and $N$ is orientable.

(4) A singularity with $g+e^{+}+e^{-}-1$ homoclinic orbits if either $e^{+}=0$ or $e^{-}=0$, but not both, and $N$ is nonorientable.

Proof. First consider the case that $N$ has genus 0 . Consider the flow on the disk with holes, $X$, depicted in Figure 4 where the number of entering and exiting holes are $e^{+}$and $e^{-}$, respectively. It is clear that the singularity $\Lambda$ in $X$ has $h_{1}=e^{+}+e^{-}-2$. Fill each of the holes of $X$ with a disk containing either a source or a sink singularity (whichever is appropriate given the flow on the corresponding boundary component). The resulting flow on the twosphere admits a three-set Morse decomposition $M_{1}, M_{2}, M_{3}$ where $M_{2}$ is the union of sources, $M_{1}$ is the singularity $\Lambda$, and $M_{0}$ is the union of the sinks. By Conley [C] and Wilson [W], there is a smooth Lyapunov function $f$ for this decomposition. If $f(\Lambda)=c$, then clearly $€>0$ can be chosen small enough such that $f^{-1}([c-\epsilon, c+\epsilon])$ is a basic block with basic set $\Lambda$ and is homeomorphic to $N$.

Now assume that $N$ is orientable and has genus $g$. Consider $X$ in Figure 4 now with $e^{+}$and $2 g+e^{-}$entering and exiting holes, respectively. The singularity $\Lambda$ in $X$ has $h_{1}=e^{+}+e^{-}+2 g-2$. Pair the $2 g$ vertical strips running between the first $2 g+1$ exiting holes, and for each pair perform the switch as depicted in Figure 5. The resulting manifold is orientable with genus $g$, has $e^{+}$entering and $e^{-}$exiting holes and the pairing has not changed $h_{1}$

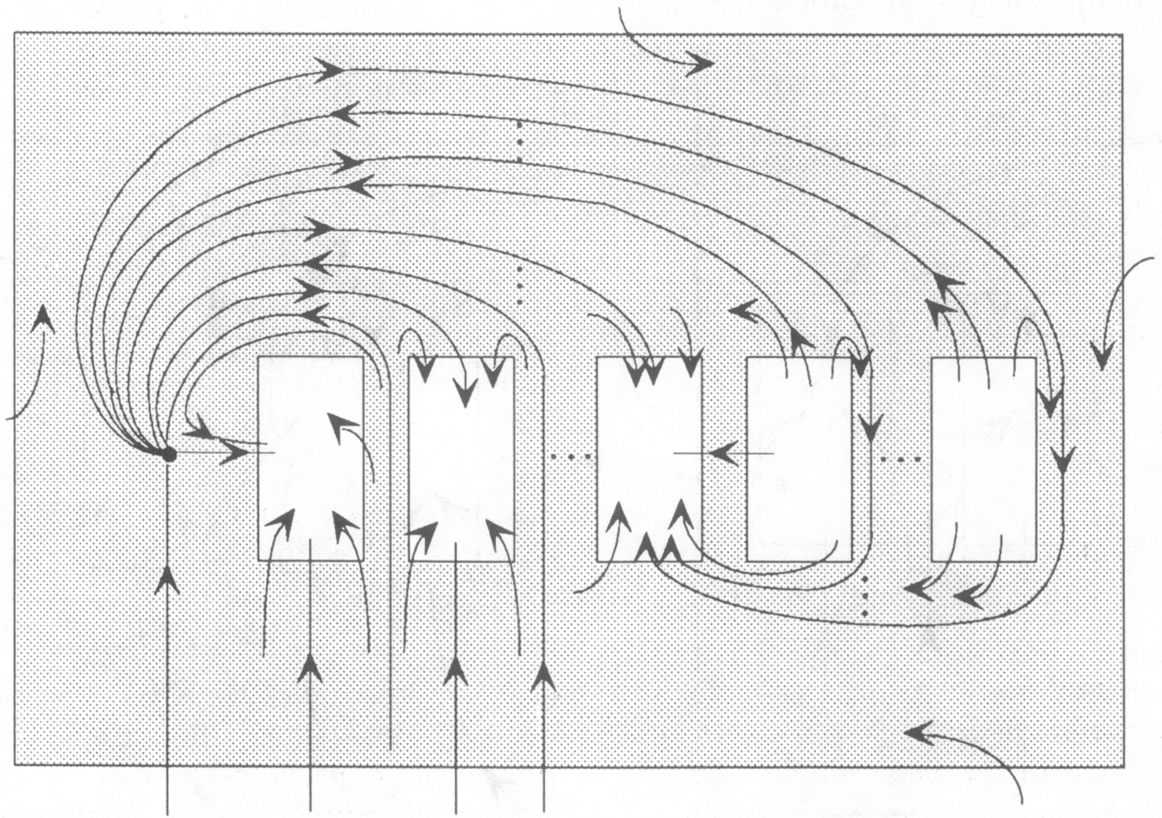

FIGURE 4. Exiting components entering components 


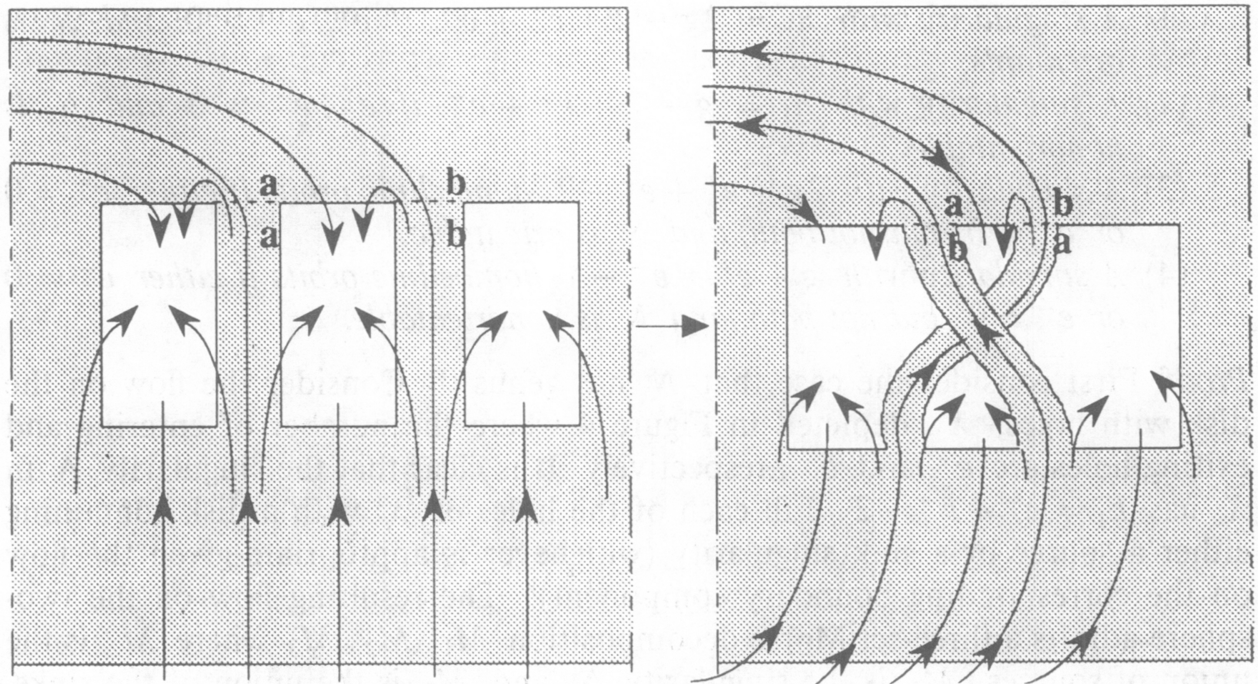

FIGURE 5. Switch strips a and b

of the singularity. As above, fill in the holes and obtain a Lyapunov function to realize $N$ as a basic block.

Now consider case (2), and assume in Figure 4 that the number of entering and exiting components of $X$ are $e^{+}$and $g+e^{-}$, respectively. The singularity $\Lambda$ in $X$ has $h_{1}=e^{+}+e^{-}+g-2$. On each of the $g$ vertical strips running between the first $g+1$ exiting holes perform the twist indicated in Figure 6 . The resulting manifold is nonorientable with genus $g$ and has $e^{+}$entering and $e^{-}$exiting holes. As above, fill in the holes and obtain a Lyapunov function to realize $N$ as a basic block.
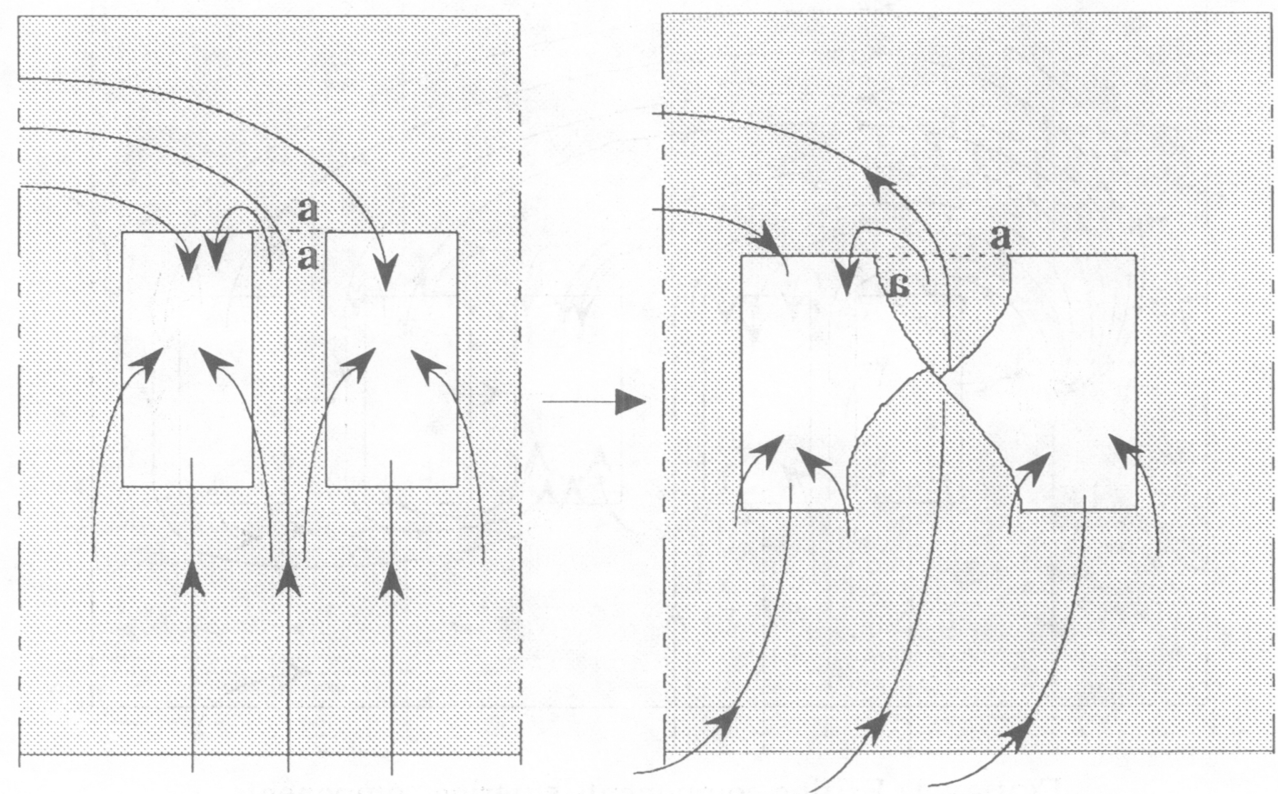

Figure 6. Twist strip a 
Cases (3) and (4) follow similar to cases (1) and (2), but, in these cases, the flow depicted in Figure 7 is used as the genus- 0 starting point if the boundary components are entering. If the boundary components are exiting then the time-reversed flow for the flow in Figure 7 is used.

Proof (Sufficiency of the local and global conditions in Theorem 2). If $\Gamma$ consists of a single vertex, then take the flow on $M$ for which every point of $M$ is a stationary point, and let $f$ be a constant function on $M$. Then $f$ is a Lyapunov function for the flow, and clearly the associated Lyapunov graph is $\Gamma$.

Now assume that $\Gamma$ consists of more than one vertex. It follows by Theorem 3 that given a vertex $v$ on an abstract Lyapunov graph $\Gamma$ labelled with homology indices $h_{0}, h_{1}$ and $h_{2}$ and of degree $b=e_{v}^{+}+e_{v}^{-}$(where $e_{v}^{+}$is the number of incoming edges and $e_{v}^{+}$is the number of outgoing edges) it can be realized as a basic block. The homology indices $h_{i}$ will determine the genus of the basic block by the formula

$$
g=\frac{h_{1}-h_{0}-h_{2}+2-b}{2}
$$

in the orientable case and by $g=h_{1}-h_{0}-h_{2}+2-b$ in the nonorientable case.

As in the proof of Theorem 1, these basic blocks can be glued together to construct a flow with a Lyapunov function whose Lyapunov graph is $\Gamma$. As in that proof some care must be taken in the gluing to ensure that the resulting

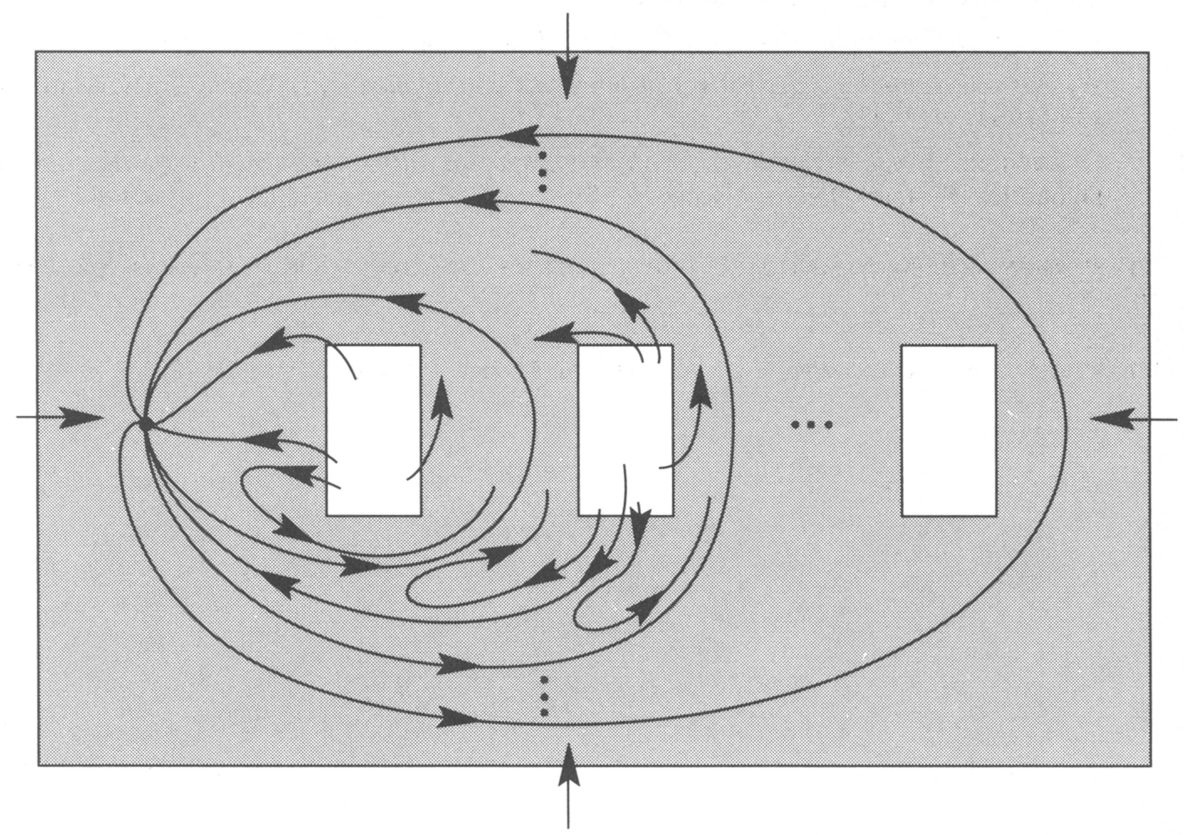

FIGURE 7. All boundary components (including the outer boundary) are entering 
manifold is (homeomorphic to) $M$. Again, the question is only one of orientation, and if the resulting manifold and $M$ are either both orientable or both nonorientable, then they are homeomorphic. Now, if $M$ is orientable, then all of the basic blocks can be chosen to be orientable, and if they are glued together preserving orientation, then the resulting manifold is orientable and thus homeomorphic to $M$. If $M$ is nonorientable and a basic block can be chosen to be nonorientable, then the resulting manifold is nonorientable and thus homeomorphic to $M$. The only situation in which $M$ is nonorientable and it is not possible to choose one of the basic blocks to be orientable is when every vertex has genus 0 . Then as in the proof of Theorem 1 , one can ensure that the resulting manifold is nonorientable (and therefore homeomorphic to $M$ ) by gluing one of the basic blocks in a way that does not preserve orientation and thereby results in a nonorientable manifold.

\section{REFERENCES}

[C] C. Conley, Isolated invariant sets and the Morse index, CBMS Regional Conf. Ser. in Math., no. 38, Amer. Math. Soc., Providence, RI, 1978.

[CZ] C. Conley and E. Zehnder, Morse type index theory for flows and periodic solutions for Hamiltonian equations, Comm. Pure Appl. Math. 37 (1984), 207-253.

[F1] J. Franks, Non-singular Smale flows on $S^{3}$, Topology 24 (1985).

[F2] _ Homology and dynamical systems, CBMS Regional Conf. Ser. in Math., no. 49, Amer. Math. Soc., Providence, RI, 1982.

[H] F. Harary, Graph theory, Addison-Wesley, Reading, Mass., 1969.

[M] J. Milnor, Morse theory, Ann. of Math. Stud., no. 51, Princeton Univ. Press, Princeton, NJ, 1963.

[P] M. Peixoto, On the classification of flows on two-manifolds, Dynamical Systems, edited by M. M. Peixoto, Academic Press, New York, 1973, pp. 389-419.

[Sm] S. Smale, Differentiable dynamical systems, Bull. Amer. Math. Soc. 73 (1967).

[W] W. Wilson, Smoothing derivatives of functions and applications, Trans. Amer. Math. Soc. 139 (1969), 413-428.

[Z] C. Zeeman, Morse inequalities for diffeomorphisms with shoes and flows with solenoids, Dynamical Systems, Lecture Notes in Math., vol. 468, Springer, 1974, pp. 44-47.

Departamento de Matemática, Universidade de Campinas, 13081 Campinas, São Paulo, BRAZIL

Department of Mathematics, University of Maine, Orono, Maine 04469 